\title{
Intraperitoneal administration of the globular adiponectin gene ameliorates diabetic nephropathy in Wistar rats
}

\author{
FANG YUAN, YING-HONG LIU, FU-YOU LIU, YOU-MING PENG and JUN-WEI TIAN \\ Department of Nephrology, The Second Xiangya Hospital, Central South University, Changsha, Hunan 410011, P.R. China
}

Received September 5, 2013; Accepted March 12, 2014

DOI: $10.3892 / \mathrm{mmr} .2014 .2133$

\begin{abstract}
The present study investigated the potential effects of the long-term expression of exogenous adiponectin (ADPN) on normal and diabetic kidneys. Type 2 diabetes mellitus models were induced by high-lipid and high-sucrose feeding plus intraperitoneal injection of streptozotocin. The recombinant plasmid pIRES2-EGFP-gAd, which is able to co-express globular ADPN (gAd) and enhanced green fluorescent protein (EGFP), was intraperitoneally injected into rat models mediated by Lipofectamine. In total, 32 Wistar rats were randomly assigned into four groups: the normal control group, the diabetes group, the diabetes group treated with pIRES2-EGFP-gAd and the diabetes group treated with pIRES2-EGFP. After 12 weeks, serum biochemistry and urine albumin levels were measured. The kidneys were collected to assess the generation of reactive oxygen species (ROS) and the renal pathological changes were observed by light microcopy. The protein expression of endothelial nitric oxide synthase (eNOS), transforming growth factor- $\beta 1$ (TGF- $\beta 1$ ) and phosphorylated adenosine monophosphate-activated protein kinase (p-AMPK) were determined by an immunohistochemical staining method and western blot analysis. Intraperitoneal injection of the human gAd gene via Lipofectamine resulted in abundant ADPN protein in the kidney. In the diabetic rats, the delivery of the exogenous gAd gene ameliorated the progression of diabetic nephropathy (DN). ADPN attenuated urine albumin excretion in the diabetic rats. ADPN also mitigated glomerular mesangial expansion, reduced the generation of ROS and prevented interstitial fibrosis. In addition, the expression of gAd inhibited the renal expression of TGF- $\beta 1$, promoted the protein expression of eNOS and activated the opening of the AMPK signaling pathway in the renal tissues of the diabetic rats. Despite the effects of ADPN on DN being
\end{abstract}

Correspondence to: Professor Ying-Hong Liu, Department of Nephrology, The Second Xiangya Hospital, Central South University, 139 Renmin Road, Changsha, Hunan 410011, P.R. China E-mail: yuanfangcat@21cn.com

Key words: diabetic nephropathy, adiponectin, urine albumin, endothelial nitric oxide synthase, transforming growth factor- $\beta 1$, adenosine monophosphate-activated protein kinase controversial, these observations indicate that the supplementation of ADPN is beneficial in ameliorating DN in rats.

\section{Introduction}

Diabetic nephropathy (DN) is the leading cause of chronic renal failure and a major long-term complication with regard to morbidity and mortality for individual diabetic patients. The pathology of DN is characterized as hypertrophy of the glomeruli, thickening of the basement membrane, accumulation of extracellular matrix (ECM) components and glomerulosclerosis. Multiple mechanisms contribute to the development of DN, including hemodynamic changes of the glomerulus, metabolic abnormalities, oxidative stress, hereditary susceptibility and inflammatory milieu. Compared with other types of kidney diseases, DN is more difficult to treat. Although the use of angiotensin-converting enzyme inhibitors and/or angiotensin receptor blockers is able to decrease the rate of DN progression (1), it is unable to prevent the occurrence of chronic renal failure. Therefore, by investigating the pathogenesis of DN, the present study focused on examining the new potential target for the treatment of DN.

Adiponectin (ADPN) is a specific secretory protein of white adipose cells that circulates in high concentrations $(\sim 0.01 \%$ of total plasma protein) and is the most abundant secretory protein of adipose tissue in human plasma. Several studies have demonstrated that hypoadiponectinemia is associated with insulin resistance (2), endothelial dysfunction (3), obesity (4), type 2 diabetes mellitus (T2DM) (5), coronary heart disease (6) and hypertension (7). Furthermore, prospective studies have demonstrated low levels to be predictive of the future risk of T2DM and myocardial infarction (6). Furthermore, complete reversal of insulin resistance in lipoatrophic mice can be achieved using by a combination of the physiological doses of ADPN and leptin, however, leptin alone causes only a partial reversal, which indicates that, as a therapeutic target, ADPN may provide a novel treatment modality for insulin resistance and T2DM (8). Due to the physiological functions of ADPN, the investigation of its association with DN has currently become a focus of study. A previous study (9) of 733 DN patients with mild to moderate renal impairment indicated that a higher level of ADPN can lead to lower chances of renal insufficiency, which indicates that a sustained high level of ADPN can slow the deterioration of renal function in patients with DN. However, Koshimura et al (10) reported 
that, for patients with diabetes mellitus, the urinary excretion and serum levels of ADPN were markedly increased in the patient group with severe renal impairment relative to the groups without renal impairment and with mild renal impairment. In addition, in patients with advanced DN, the urinary and serum ADPN levels were positively correlated. Another study (11) indicated that the serum ADPN levels were decreased in diabetic patients, but increased in the DN patients at stages 3-5. Whether or not this increase reflects impaired ADPN clearance by the kidney or whether it is a compensatory mechanism aimed at counteracting increased cardiovascular risk factors is not yet elucidated.

Although a previously reported study (12) has confirmed that ADPN may reduce proteinuria and improve kidney podocyte function for ADPN-knockout $\left(\mathrm{Ad}^{-/-}\right)$mice, it remains unclear whether this role of ADPN is associated with the special genetic background and gene variation of $\mathrm{Ad}^{-/}$mice or not. Given that the role of ADPN in the early stage of DN is not clear, the present study aimed to investigate the effects of ADPN on the kidneys of streptozotocin (STZ)-induced diabetic rats and to examine its possible mechanisms.

\section{Materials and methods}

Animals. The animal study was conducted in accordance with the protocols approved by the experimental animal ethics committee of Central South University (Changsha, Hunan, China). A total of 328 -week-old male Wistar rats, with a mean body weight of $202 \pm 3 \mathrm{~g}$ and bred in the standard environment with free access to standard feed and drinking tap water, were provided by the animal lab of Central South University (Changsha, Hunan, China). For evaluating the effects of ADPN on diabetic kidneys, a diabetic model was used. The rats were fed a high-sucrose/fat diet (consisting of $10 \%$ lard, $20 \%$ sucrose, $2.5 \%$ cholesterol, $1 \%$ bile salt and $66.5 \%$ general food) for 1 month. Following induction of insulin resistance, a low dose $(30 \mathrm{mg} / \mathrm{kg})$ of $1 \%$ STZ (Sigma, St. Louis, MO, USA) was administered by intraperitoneal injection (13). After $72 \mathrm{~h}$, the blood glucose (BG) levels were measured and found to be consistently higher than $16.7 \mathrm{mmol} / \mathrm{l}$, accompanied by the symptoms of excess thirst, frequent urination, constant hunger and weight loss, which indicated that the T2DM model had been established successfully.

After 1 week, the Wistar rats were randomly divided into four groups of eight rats each as follows: i) Normal control group, which was fed regular food; ii) diabetic group without any therapy; iii) diabetic group treated with pIRES2-EGFP-gAd [the fluorescence plasmid of pIRES2-EGFP-gAd containing a full-length coding region of the globular domain of ADPN, and which can co-express globular ADPN protein (gAd) and enhanced green fluorescent protein (EGFP), was constructed and stored by the Nephrology Laboratory of The Second Xiangya Hospital (Changsha, Hunan, China)]. The recombinant plasmid, plRES2-EGFP-gAd, was intraperitoneally injected into the DM rats mediated by Lipofectamine $(1 \mu \mathrm{g}: 0.5 \mu \mathrm{l}$ of plasmid) with a $200 \mu \mathrm{g} / \mathrm{kg}$ dosage to body weight twice a week; and iv) diabetic group treated with pIRES2-EGFP. The recombinant plasmid, plRES2-EGFP, was intraperitoneally injected in the DM rats mediated by Lipofectamine (1 $\mu \mathrm{g}: 0.5 \mu \mathrm{g}$ of plasmid) with $200 \mu \mathrm{g} / \mathrm{kg}$ dosage to body weight twice a week. Urine was collected for total protein and albumin determination at 4,8 and 12 weeks after the injection of STZ. At 4 and 12 weeks after the administration of STZ, the rats were euthanized by $\mathrm{CO}_{2}$ inhalation and the serum, urine and kidneys were analyzed as described next.

Additional groups of rats were also used to study exogenous gAd expression at the different time-points indicated following a single injection of gAd plasmid.

Determination of gAd expression by fluorescence microscopy and western blot analysis. Once injected with pIRES2-EGFP-gAd, the rats were sacrificed after 24,48 or $96 \mathrm{~h}$ or after 7 days, and the frozen sections of kidneys were used to observe the expression of GFP in renal tissue using fluorescence microscopy. The extracted protein in the renal tissues of the rats was hybridized with anti-ADPN (mouse monoclonal antibody; EMD Millipore, Billerica, MA, USA; $30 \mathrm{KD}$ ) and $\beta$-actin antibodies (mouse monoclonal antibody; Sigma) and colored using an enhanced chemiluminescence method. A semi-quantitative analysis was performed using a UVP gel image system (Bio-Rad, Hercules, CA, USA) to determine ADPN expression in the renal tissue.

Determination of transforming growth factor- $\beta 1$ (TGF- $\beta 1$ ) levels by ELISA. The TGF- $\beta 1$ levels in the serum and urine were determined using a commercial Quantikine TGF- $\beta 1$ ELISA kit, according to the manufacturer's instructions (R\&D Systems, Minneapolis, MN, USA). This kit detects active TGF- $\beta 1$ protein that binds to its soluble type II receptor precoated onto a microplate. The TGF- $\beta 1$ levels in the serum and urine were expressed as $\mathrm{pg} / \mathrm{ml}$ and $\mathrm{ng} / \mathrm{mg}$ creatinine, respectively.

Morphological studies. Kidney sections were prepared at a thickness of $3 \mu \mathrm{m}$ by a routine procedure, stained with hematoxylin and eosin and Masson trichrome, observed with light microscopy and analyzed with a medical image analysis system (Northern Navigation Co., Ltd., Stamford, CT, USA) to quantitatively analyze the morphology of the glomeruli.

Western blot analysis and immunohistochemical staining. The protein expression in the kidney tissue was analyzed by western blot analysis and an immunohistochemical staining method. The primary antibodies used were as follows: Anti-endothelial nitric oxide synthase (eNOS; mouse monoclonal; Abcam, Cambridge, MA, USA), anti-TGF- $\beta 1$ (mouse monoclonal; Abcam), anti-adenosine monophosphate-activated protein kinase (AMPK), anti-phospho-AMPK- $\alpha$ (rabbit polyclonal antibodies; Cell Signaling Technology, Danvers, MA, USA) and anti- $\beta$-actin (Sigma).

Determination of reactive oxygen species (ROS) levels in the kidney tissues. Kidney tissue samples were prepared by mixing kidney tissue with $0.9 \%$ saline solution to obtain $10 \%$ uniform slurry and measured according to the manufacturer's instructions (Nanjing Jiancheng Bioengineering Institute, Nanjing, Jiangsu, China) to detect the ROS levels in the organization.

Serum biochemistry. The serum concentrations of total protein, albumin, alanine aminotransferase, blood urea nitrogen and 
serum creatinine were measured by an automated analyzer in the clinical chemistry laboratory of The Second Xiangya Hospital.

Determination of $B G$, creatinine and albumin. The levels of BG were determined using the Accu-Chek Active glucometer and test strips (Roche Diagnostics, Indianapolis, IN, USA). The serum and urine creatinine levels were determined by a routine procedure. The total protein levels were determined using a bicinchoninic acid-based protein assay kit (Sigma) with bovine serum albumin as a standard. Urine albumin was measured using a mouse Albumin ELISA Quantitation kit, according to the manufacturer's instructions (Bethyl Laboratories, Inc., Montgomery, TX, USA).

Statistical analysis. A statistical analysis of the data was performed using SPSS 12.0 software (SPSS, Inc., Chicago, IL, USA). The comparison between groups was conducted using a one-way analysis of variance followed by the Student-Newman-Keuls test. $\mathrm{P}<0.05$ was considered to indicate a statistically significant difference.

\section{Results}

Renal expression of the EGFP reporter gene and ADPN protein. The results of the frozen tissue sections are shown in Fig. 1A. The expression of GFP could be observed in the glomeruli and renal interstitium using fluorescence microscopy $24 \mathrm{~h}$ after intraperitoneal injection with the recombinant pIRES2-EGFP-gAd plasmid with liposome, and after $48 \mathrm{~h}$, the intensity was enhanced, particularly in the renal tubules. However, with increasing time following the injection, the expression of GFP in the kidney gradually decreased; 7 days after injections, although the expression of GFP could still be detected, the fluorescence intensity had decreased significantly. In addition, no GFP expression was detected for the samples from the normal control group. The results indicated that the kidney was successfully transfected with the plasmid.

Fig. 1B shows the human gAd protein levels in the renal tissue of additional groups of rats at the different time-points following a single plasmid injection, as detected by western blot analysis using an anti-ADPN antibody against gAd. The results were consistent with the aforementioned detection of the reporter gene and showed that there was abundant exogenous gAd protein in the kidney following intraperitoneal injection of the plasmid vector, which varied over time.

Effects of sustained ADPN expression on normal kidneys. The present study examined the potential effects of the sustained expression of exogenous gAd on normal kidneys. To achieve long-term expression, the pIRES2-EGFP-gAd plasmid was repeatedly administered to the rats using biweekly intraperitoneal injections. Thus, two groups of normal rats received injections of either pIRES2-EGFP or pIRES2-EGFP-gAd twice a week for 8 weeks. No gross or microscopic alterations were identified in the kidney morphology between these two groups at the end of week 8 . Table I shows the data on serum biochemistry, urine protein excretion and renal TGF- $\beta 1$ levels in the rats that received biweekly plasmid injections for 8 weeks. No significant difference in blood urea nitrogen, creatinine and urine protein excretion was identified between these two groups $(P>0.05 ; n=6)$. Furthermore, the renal TGF- $\beta 1$ levels were similar in the rats that received either pIRES2-EGFP or pIRES2-EGFP-gAd injections for 8 weeks (Table I).

$A D P N$ ameliorates proteinuria in $D N$. The present study next investigated the effects of ADPN on the progression of DN. Table II shows the general characteristics of diabetic rats at the end of 4 and 12 weeks after STZ injection. Two out of eight rats died over the 12 -week period in the diabetic group and one out of the eight rats died in the group that received the injection of pIRES2-EGFP (Table II). A substantial reduction in weight gain was observed in the diabetic rats over the 12-week period compared with the diabetic group (Table II). The intraperitoneal injection of the gAd plasmid was able to decrease the BG levels in the surviving animals when administered 4 and 12 weeks after STZ injection (Table II). The diabetic kidney at 4 and 12 weeks post-injection with STZ exhibited marked enlargement in size, which was significantly reduced by ADPN (Table II). However, the serum creatinine level only slightly increased in the diabetic rats 12 weeks after STZ injection compared with that in the normal controls (Table II). No statistical difference was identified in the general characteristics between the pIRES2-EGFP group and the diabetic group.

The expression of exogenous ADPN resulted in a substantial alleviation of proteinuria in the diabetic rats. Fig. 2 shows the urine albumin levels at the different time points in the diabetic rats. At 12 week after STZ injection, the urine albumin level reached $1.5 \mathrm{mg} / \mathrm{mg}$ creatinine. However, the injection of the $\mathrm{gAd}$ plasmid reduced the level of albumin excretion by $>70 \%$ (Fig. 2).

ADPN attenuates mesangial expansion and matrix deposition in the glomeruli. Using a light microscope, the stained kidney tissue structure was normal in the control group. Compared with the control group, there was glomerular hypertrophy, mesangial expansion, basement membrane thickening, tubular epithelial cell cavitation and exfoliation and mononuclear lymphocyte infiltration in the DM group, all of which were consistent with the changes in DN (Fig. 3B and F). However, these changes were ameliorated in the gAd transfection group (Fig. 3C and G). Using light microscopy, no clear difference was observed between the DM and the pIRES2-EGFP groups. The quantitative analysis of morphology is shown in Table III.

$A D P N$ reduces the generation of $R O S$ in the kidneys of DM rats. After 12 weeks, the amount of generated ROS in the DM, gAd transfection and pIRES2-EGFP groups was significantly greater than that in the normal control group $(\mathrm{P}<0.05)$, while the amount of generated ROS in the gAd transfection group was less than that in the DM group $(\mathrm{P}<0.05)$. In addition, no significant difference was identified in the amount of generated ROS between the DM group and the pIRES2-EGFP group (Table II). The present study indicated that ADPN could reduce the generation of ROS in the renal tissues of rats with DN. 
Table I. Serum biochemistry, urine protein excretion and kidney TGF- $\beta 1$ levels in rats that received biweekly plasmid injections for 8 weeks.

\begin{tabular}{|c|c|c|c|c|c|c|c|}
\hline \multirow[b]{2}{*}{ Plasmid } & \multicolumn{5}{|c|}{ Serum biochemistry } & \multirow{2}{*}{$\begin{array}{l}\text { Urine protein } \\
\text { excretion, } \\
\mathrm{mg} / \mathrm{mg} \mathrm{Cr}\end{array}$} & \multirow{2}{*}{$\begin{array}{c}\text { Kidney } \\
\text { TGF- } \beta 1, \\
\text { pg/mg } \\
\text { protein }\end{array}$} \\
\hline & $\begin{array}{l}\text { Total protein, } \\
\text { g/dl }\end{array}$ & $\begin{array}{l}\text { Albumin, } \\
\mathrm{g} / \mathrm{dl}\end{array}$ & $\begin{array}{l}\mathrm{ALT}, \\
\mathrm{U} / 1\end{array}$ & $\begin{array}{l}\text { BUN, } \\
\mathrm{mmol} / \mathrm{l}\end{array}$ & $\begin{array}{c}\text { Creatinine, } \\
\qquad \mu \mathrm{mol} / 1\end{array}$ & & \\
\hline pIRES2-EGFP & $4.85 \pm 0.11$ & $2.12 \pm 0.03$ & $11 \pm 0.13$ & $9.94 \pm 1.86$ & $19.64 \pm 5.36$ & $89 \pm 11.0$ & $9.82 \pm 2.58$ \\
\hline pIRES2-EGFP-gAd & $5.06 \pm 0.21$ & $2.23 \pm 0.05$ & $9.30 \pm 0.11$ & $8.52 \pm 0.65$ & $18.29 \pm 4.15$ & $90 \pm 7.0$ & $8.98 \pm 3.68$ \\
\hline
\end{tabular}

ALT, alanine aminotransferase; BUN, blood urea nitrogen; Cr, creatinine; TGF- $\beta 1$, transforming growth factor- $\beta 1$; EGFP, enhanced green fluorescent protein; gAd, globular adiponectin. No statistical difference $(\mathrm{P}>0.05 ; \mathrm{n}=6)$ was identified in all parameters between these two groups.

Table II. General characteristics of mice among different treatment groups.

\begin{tabular}{|c|c|c|c|c|c|c|c|}
\hline \multirow[b]{2}{*}{ Characteristic } & \multirow{2}{*}{$\frac{\text { Normal control }}{12 \text { weeks }}$} & \multicolumn{2}{|c|}{ Diabetic rats } & \multicolumn{2}{|c|}{ STZ + pIRES2-EGFP-gAd } & \multicolumn{2}{|c|}{$\mathrm{STZ}+$ pIRES2-EGFP } \\
\hline & & 4 weeks & 12 weeks & 4 weeks & 12 weeks & 4 weeks & 12 weeks \\
\hline Animal numbers & $8 / 8$ & & $8 / 6$ & & $8 / 8$ & & $8 / 7$ \\
\hline Body weight, g & $202 \pm 3$ & $171 \pm 2^{\mathrm{a}}$ & $167 \pm 4^{\mathrm{a}}$ & $180 \pm 3^{\mathrm{a}}$ & $179 \pm 2^{\mathrm{a}}$ & $170 \pm 1^{\mathrm{a}}$ & $165 \pm 3^{\mathrm{a}}$ \\
\hline $\mathrm{BG}, \mathrm{mmol} / \mathrm{l}$ & $5.7 \pm 1.1$ & $24.7 \pm 3.12^{\mathrm{a}}$ & $25.8 \pm 2.2^{\mathrm{a}}$ & $19.5 \pm 4.7^{\mathrm{a}, \mathrm{b}}$ & $15.3 \pm 2.1^{\mathrm{a}, \mathrm{b}}$ & $24.3 \pm 2.1^{\mathrm{a}}$ & $26.4 \pm 1.1^{\mathrm{a}}$ \\
\hline $\mathrm{KW} / \mathrm{BW}\left(\mathrm{x} 10^{-3}\right)$ & $7.0 \pm 1.2$ & $19.6 \pm 0.7^{\mathrm{a}}$ & $21.3 \pm 2.9^{\mathrm{a}}$ & $18.0 \pm 0.8^{\mathrm{a}}$ & $16.1 \pm 0.6^{\mathrm{a}, \mathrm{b}}$ & $19.4 \pm 0.9^{\mathrm{a}}$ & $20.2 \pm 0.4^{\mathrm{a}}$ \\
\hline Serum creatinine, $\mu \mathrm{mol} / 1$ & $18.3 \pm 4.2$ & $\mathrm{nt}$ & $25.0 \pm 4.8^{\mathrm{a}}$ & nt & $20.6 \pm 5.4$ & nt & $21.7 \pm 7.4$ \\
\hline $\mathrm{ROS}, \mathrm{nmol} / \mathrm{l}$ & $15.7 \pm 4.1$ & $\mathrm{nt}$ & $29.1 \pm 2.2^{\mathrm{a}}$ & nt & $20.1 \pm 1.6^{\mathrm{a}, \mathrm{b}}$ & nt & $28.8 \pm 3.1^{\mathrm{a}}$ \\
\hline
\end{tabular}

Animal numbers are presented as number at beginning of experiment/number at end of experiment. Serum creatinine levels and KW/BW measurements were obtained at the end of the experiment. KW, kidney weight; BW, body weight; STZ, streptozotocin; nt, not tested; EGFP, enhanced green fluorescent protein; gAd, globular adiponectin; ROS, reactive oxygen species; BG, blood glucose. ${ }^{\text {a }}<0.05$ vs. normal controls . ${ }^{\mathrm{b}} \mathrm{P}<0.05$ vs. diabetic rats.

Table III. Quantitative morphological analysis in rat kidneys (mean \pm standard deviation).

\begin{tabular}{lcccc}
\hline Group & $\mathrm{n}$ & $\begin{array}{c}\text { Glomerular sectional area } \\
\left(\mu \mathrm{m}^{2}\right)\end{array}$ & $\begin{array}{c}\text { Glomerular matrix area } \\
\left(\mu \mathrm{m}^{2}\right)\end{array}$ & $\begin{array}{c}\text { Matrix area/sectional area } \\
\left(\mu \mathrm{m}^{2} / \mu \mathrm{m}^{2}\right)\end{array}$ \\
\hline Normal control & 8 & $37571.05 \pm 2320.58$ & $937.02 \pm 416.31$ & $0.025 \pm 0.011$ \\
Diabetic rats & 6 & $42469.17 \pm 6132.50^{\mathrm{a}}$ & $1962.93 \pm 578.70^{\mathrm{a}}$ & $0.046 \pm 0.015^{\mathrm{a}}$ \\
STZ + pIRES2-EGFP-gAd & 8 & $40022.77 \pm 2551.62^{\mathrm{a}}$ & $1435.53 \pm 399.97^{\mathrm{a}, \mathrm{b}}$ & $0.036 \pm 0.010^{\mathrm{a}}$ \\
STZ + pIRES2-EGFP & 7 & $44981.36 \pm 2029.74^{\mathrm{a}}$ & $1608.92 \pm 350.28^{\mathrm{a}}$ & $0.040 \pm 0.009^{\mathrm{a}}$
\end{tabular}

${ }^{\mathrm{a}} \mathrm{P}<0.05$ vs. normal controls; ${ }^{\mathrm{b}} \mathrm{P}<0.05$ vs. diabetic rats. EGFP, enhanced green fluorescent protein; gAd, globular adiponectin; STZ, streptozotocin.

gAd promotes the protein expression of eNOS in the renal tissue of rats. In the normal control group (Fig. 4A), the protein expression of eNOS was identified in the rat kidney, and immunohistochemical analysis demonstrated brown-yellow granules. In the DM group (Fig. 4B), the protein expression of eNOS was significantly lower than that in the control group $(\mathrm{P}<0.05)$, and in the gAd transfection group, although the expression of eNOS remained lower than that in the control group, it was significantly higher than that in the DM group $(\mathrm{P}<0.05)$. The protein expression of eNOS was mainly observed in the mesangial area, basement membrane, cytoplasm of renal tubular epithelial cells and renal intersti- tium. Western blot analysis (Fig. 4E) also indicated that the protein level of eNOS decreased by $285 \%$ in the renal tissues of the rats in the DM group compared with that in the control group $(\mathrm{P}<0.05)$, and that the level increased by $221 \%$ in the gAd transfection group compared with that in the DM group $(\mathrm{P}<0.05)$, which indicated that ADPN could enhance the protein expression of eNOS in the renal tissues of diabetic rats.

gAd inhibits TGF- $\beta 1$ expression in DN. The expression of TGF- $\beta 1$ was detected in the rat kidney of the normal control group and was significantly lower than that in the DM group, 
Table IV. Comparison of eNOS, TGF- $\beta 1$ and p-AMPK immunohistochemical average optical density of each group (mean \pm standard deviation).

\begin{tabular}{lllll}
\hline Group & $\mathrm{n}$ & eNOS, $\%$ & TGF- $\beta 1, \%$ & AMPK, $\%$ \\
\hline Normal control & 8 & $1.56 \pm 0.41$ & $0.73 \pm 0.41$ & $2.08 \pm 0.84$ \\
Diabetic rats & 6 & $0.56 \pm 0.47^{\mathrm{a}}$ & $2.59 \pm 0.57^{\mathrm{a}}$ & $1.97 \pm 0.98$ \\
STZ + pIRES2-EGFP-gAd & 8 & $1.48 \pm 0.66^{\mathrm{b}}$ & $0.98 \pm 0.50^{\mathrm{a}, \mathrm{b}}$ & $3.69 \pm 1.59^{\mathrm{a}, \mathrm{b}}$ \\
STZ + pIRES2-EGFP & 7 & $0.58 \pm 0.50^{\mathrm{a}}$ & $2.48 \pm 0.66^{\mathrm{a}}$ & $1.99 \pm 0.52$ \\
\hline
\end{tabular}

${ }^{\mathrm{a}} \mathrm{P}<0.05$ vs. normal controls; ${ }^{\mathrm{b}} \mathrm{P}<0.05$ vs. diabetic rats. EGFP, enhanced green fluorescent protein; gAd, globular adiponectin; eNOS, endothelial nitric oxide synthase; TGF- $\beta 1$, transforming growth factor- $\beta 1$; p-AMPK, phosphorylated AMP-activated protein kinase; STZ, streptozotocin.

A
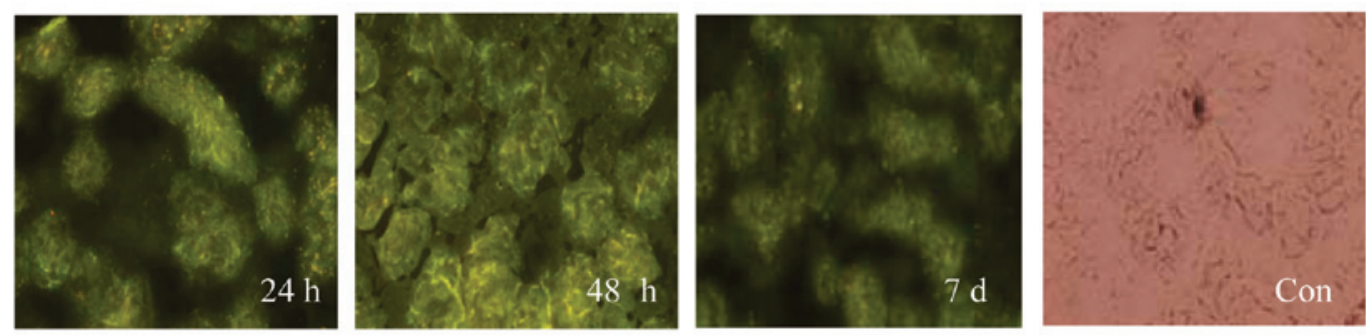

B

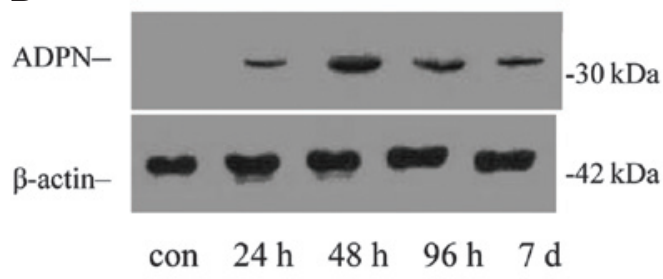

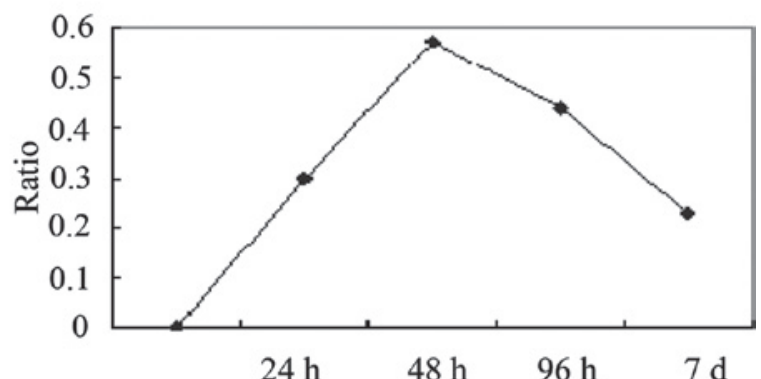

Figure 1. Expression of the ADPN protein in rat kidneys following intraperitoneal injection of the pIRES2-EGFP-gAd plasmid. (A) Following $24 \mathrm{~h}$ of the intraperitoneal injection of pIRES2-EGFP-gAd, the expression of GFP could be observed in the glomeruli and tubules using a fluorescence microscope, and following $48 \mathrm{~h}$, the intensity was enhanced, particularly in the renal tubule. With increasing time following the injection, the expression of GFP gradually reduced, which indicated that the plasmid had successfully transfected into the kidney. (B) Western blot analysis shows the levels of ADPN protein were consistent with the detection of the EGFP reporter gene in the rat kidney following intraperitoneal injection of the pIRES2-EGFP-gAd plasmid. ADPN, adiponectin; EGFP, enhanced green fluorescent protein; gAd, globular adiponectin; con, control.

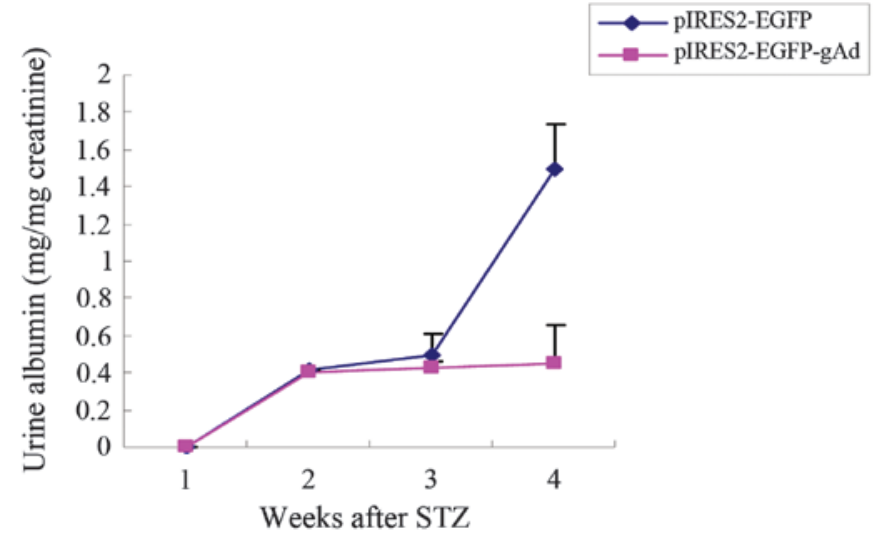

Figure 2. ADPN ameliorates proteinuria in diabetic mice. Urine albumin was determined at different time-points, as indicated, in diabetic mice that received pIRES2-EGFP or pIRES2-EGFP-gAd plasmid injections. Data are expressed as $\mathrm{mg} / \mathrm{mg}$ urine creatinine and presented as the mean \pm standard error of the mean of six to eight animals per group. ${ }^{a} \mathrm{P}<0.05$ vs. pIRES2-EGFP group. ADPN, adiponectin; GFP, green fluorescent protein; gAd, globular adiponectin; STZ, streptozotocin.
gAd transfection or pIRES2-EGFP groups $(\mathrm{P}<0.05)$. The level was also significantly lower in the gAd transfection group compared with that in the DM or pIRES2-EGFP groups $(\mathrm{P}<0.05)$, which indicated that the expression of TGF- $\beta 1$ protein in the kidney of DM rats could be inhibited by ADPN. The results are shown in Fig. 5.

Expression of $p-A M P K$ in the renal tissue of rats. The results of immunohistochemical staining and western blot analysis (Fig. 6) verified that p-AMPK was expressed in the normal rat kidney. Fig. 6A shows AMPK activation in the renal tissue following a single injection of the gAd plasmid, which indicated that ADPN was able to induce the opening of the AMPK channel in a time-dependent manner. The results of the UVP gel image scanner quantitative analysis demonstrated that following $16 \mathrm{~h}$ of activation by ADPN the expression of the p-AMPK protein was the strongest, without any changes in the total amount of AMPK protein. At the end of the test, the expression of p-AMPK was significantly 

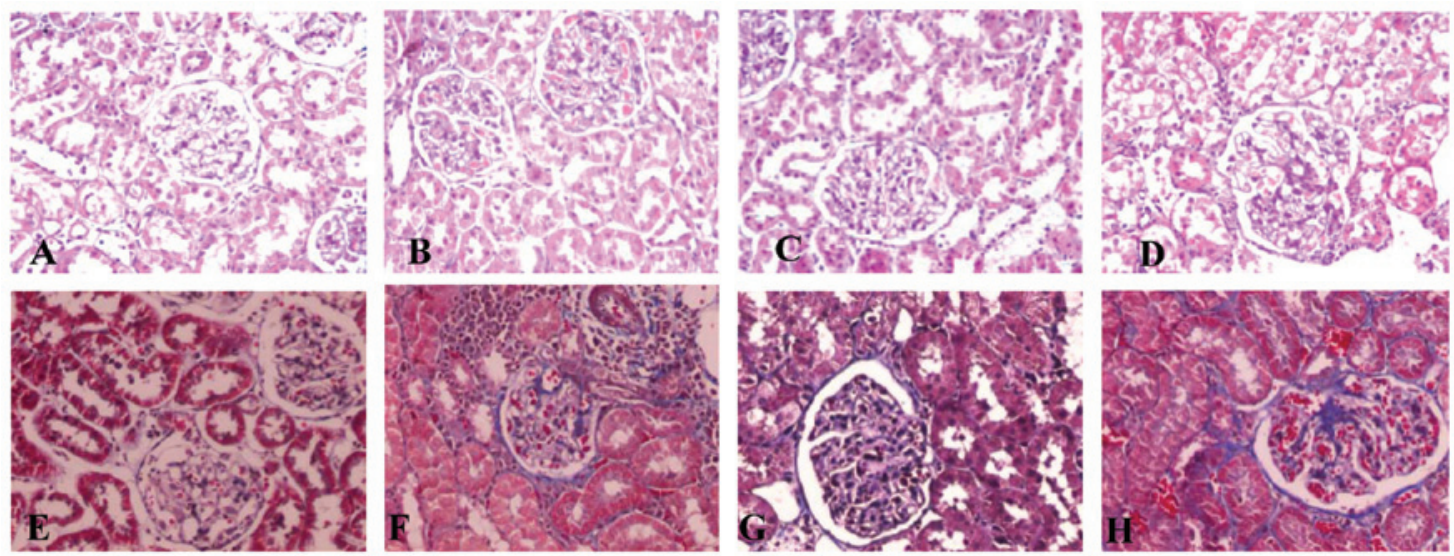

Figure 3. ADPN reduces mesangial expansion and attenuates glomerular matrix accumulation in DN. (A-D) Representative micrographs demonstrating mesangial expansion, morphological injury (H\&E staining) and (E-H) glomerular collagen deposition (Masson-trichrome staining) in (A and E) normal control rats, $(\mathrm{B}$ and $\mathrm{F})$ diabetic rats, $(\mathrm{C}$ and $\mathrm{G})$ diabetic rats that received pIRES2-EGFP-gAd injections and (D and $\mathrm{H})$ diabetic rats that received pIRES2-EGFP injections. ADPN, adiponectin; DN, diabetic nephropathy; H\&E, hematoxylin and eosin; EGFP, enhanced green fluorescent protein; gAd, globular adiponectin.
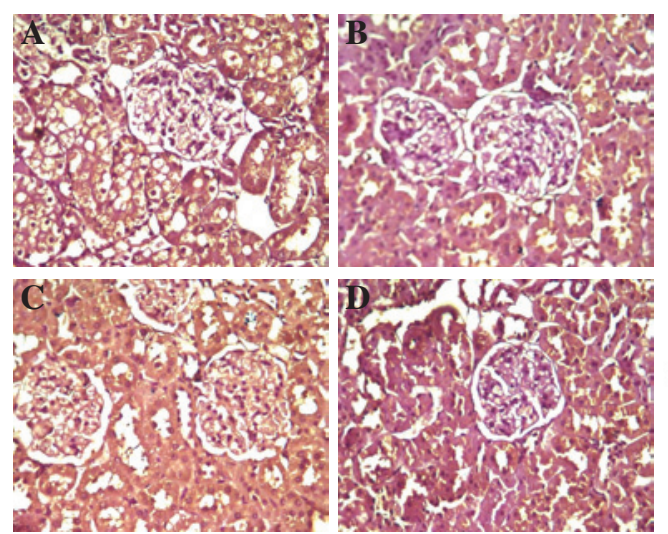

$\mathbf{E}$

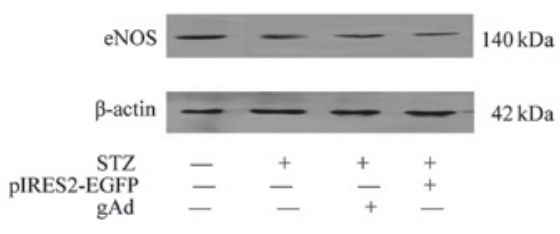

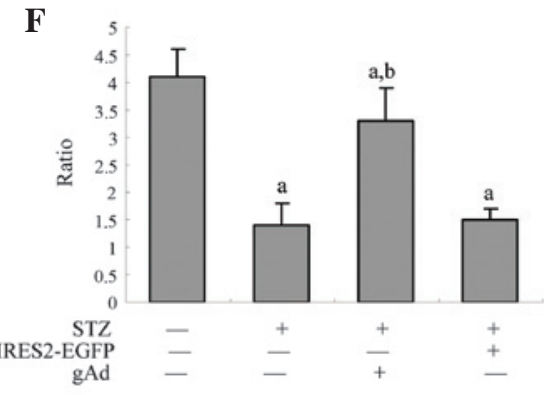

Figure 4. ADPN stimulates eNOS activation in diabetic kidneys. (A-D) Representative micrographs demonstrating the localization of eNOS (brown-yellow granules) in normal and diabetic kidneys. (A) Normal control. (B) Diabetic kidney. (C) Diabetic kidney with pIRES2-EGFP-gAd (D) Diabetic kidney with pIRES2-EGFP. (E and F) Representative western blot analysis and graphical presentation demonstrating the effects of ADPN on eNOS expression in diabetic kidneys. Whole-tissue homogenates were subjected to western blot analysis using antibodies against eNOS and $\beta$-actin. Data in (F) are presented as the mean \pm standard error of the mean of six animals per group. ${ }^{\mathrm{P}} \mathrm{P}<0.05$ vs. normal controls; ${ }^{\mathrm{b}} \mathrm{P}<0.05$ vs. diabetic rats. ADPN, adiponectin; eNOS, endothelial nitric oxide synthase; EGFP, enhanced green fluorescent protein; gAd, globular adiponectin; STZ, streptozotocin.
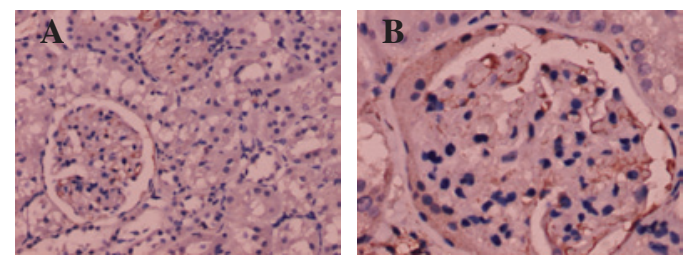

$\mathbf{E}$

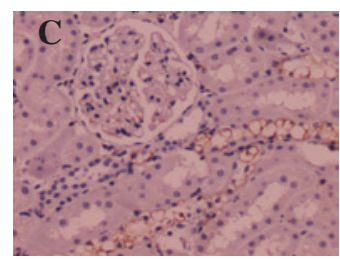

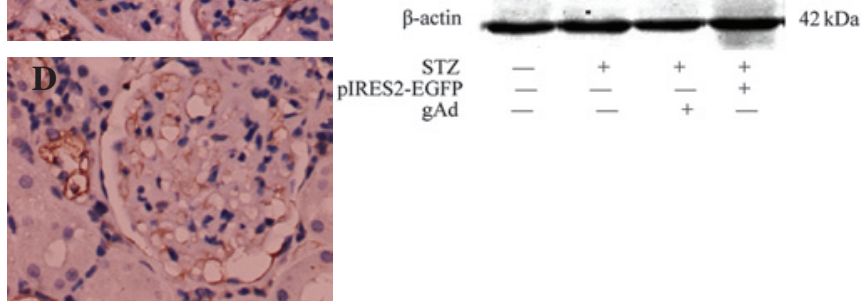

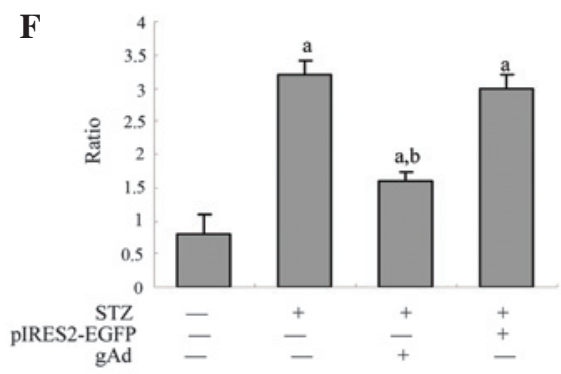

Figure 5. ADPN inhibits TGF- $\beta 1$ activation in diabetic kidneys. (A-D) Representative micrographs demonstrating the localization of TGF- $\beta 1$ (brown-yellow granules) in normal and diabetic kidneys. (A) Normal control. (B) Diabetic kidney. (C) Diabetic kidney with pIRES2-EGFP-gAd (D) Diabetic kidney with pIRES2-EGFP. (E and F) Representative western blot analysis and graphical presentation demonstrating the effects of ADPN on TGF- $\beta 1$ expression in diabetic kidneys. Data in $(\mathrm{F})$ are presented as the mean \pm standard error of the mean of six animals per group. ${ }^{a} \mathrm{P}<0.05$ vs. normal controls; ${ }^{\mathrm{b}} \mathrm{P}<0.05$ vs. diabetic rats. ADPN, adiponectin; transforming growth factor- $\beta 1$; EGFP, enhanced green fluorescent protein; gAd, globular adiponectin; STZ, streptozotocin. 
A

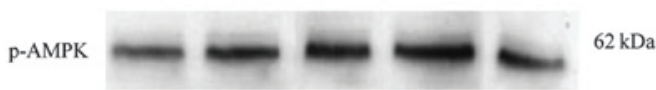

AMPK $62 \mathrm{kDa}$
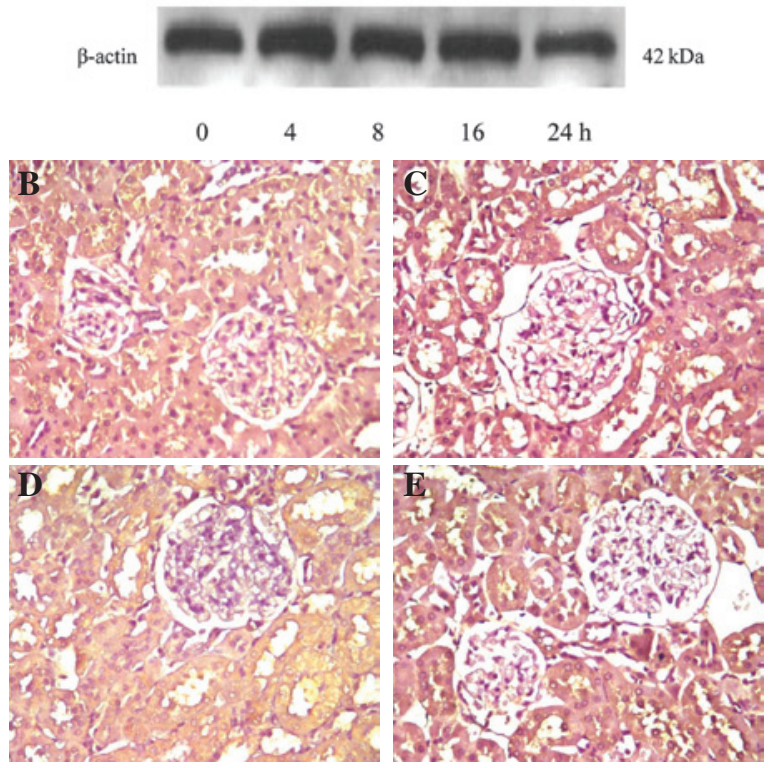

Figure 6. ADPN stimulates AMPK activation in diabetic kidneys. (A) AMPK activation in the renal tissue following a single injection of gAd plasmid. (B-E) Representative micrographs demonstrating the localization of AMPK in normal and diabetic kidneys by immunohistochemical staining. (A) Normal control. (B) Diabetic kidney. (C) Diabetic kidney with pIRES2-EGFP-gAd (D) Diabetic kidney with pIRES2-EGFP. ADPN, adiponectin; EGFP, enhanced green fluorescent protein; gAd, globular adiponectin; p-AMPK phosphorylated AMP-activated protein kinase.

higher in the gAd transfection group compared with that in the control and DM groups $(\mathrm{P}<0.05)$, and that it was lower in the DM group compared with that in the normal control group $(\mathrm{P}<0.05)$. The results indicated that ADPN could activate the opening of the AMPK signaling pathway in the renal tissues of diabetic rats.

Correlation analysis. The average optical density analysis demonstrated that eNOS and p-AMPK were positively correlated (Table IV; $\mathrm{r}=0.685 ; \mathrm{P}<0.05$ ).

\section{Discussion}

There are numerous types of animal models for investigating diabetes and its complications. In the present study, a traditional model for T2DM was successfully constructed using low dose injection of STZ and high-sucrose/fat feeds. Through use of intraperitoneal injections of the recombinant pIRES2-EGFP-gAd plasmid, the ADPN gene was successfully transfected into the rat kidney.

The present study demonstrated that the sustained expression of exogenous gAd in the kidney did not cause adverse side-effects on normal renal structure and function. Furthermore, the expression of the gAd gene prevented the onset and progression of DN, resulting in reduced proteinuria, attenuated glomerulosclerotic lesions and decreased renal TGF- $\beta 1$ expression. These observations are consistent with several previous studies demonstrating that ADPN is a protective factor in a wide variety of chronic kidney diseases (14) and indicate that supplementation with exogenous ADPN may be an effective strategy for the prevention and treatment of DN.

$\mathrm{DN}$ is one of the major chronic complications of type $1 \mathrm{DM}$ (T1DM) and T2DM, and also one of the leading causes of mortality in patients with T1DM. Multiple mechanisms contributed to the development and outcomes of DN, including an interaction between hyperglycemia-induced metabolic and hemodynamic changes and genetic predisposition, which increase susceptibility to kidney injury. Clinical study and animal experiments (15) indicated that in numerous pathologies, oxidative stress was an important common mechanism. In the case of diabetes it is often accompanied by increases in ROS or decreases in the function of the antioxidant enzyme system, resulting in disruption of the balance between the generation and clearance of ROS in the local renal tissue. Obrosova et al (16) reported that the content of the antioxidant $\mathrm{Vit} \mathrm{C}$ in the kidney tissue of diabetic rats was significantly lower than that in the normal rats, indicating that with DM, the 'use' of Vit C in renal tissue decreased. Excess ROS accumulating in the body is able to activate signal transduction cascade pathways and transcription factors, which can damage intracellular proteins, and activate lipid peroxidation in liposome membranes or DNA, causing cell death and tissue damage. In addition, high BG levels in diabetic patients can cause increased TGF- $\beta 1$ expression, and TGF- $\beta 1$ is an important cytokine in early or late DN $(17,18)$. In a previous study, TGF- $\beta 1$ mediated the renal mesangial cells, endothelial cells and fibroblasts to produce excessive ECM (18), and was also able to reduce degradation of the ECM by reducing the expression of matrix metalloproteinases and promoting the synthesis of plasminogen activator inhibitor and tissue inhibitors of metalloproteinases, resulting in a large amount of ECM deposition in the renal interstitium, eventually leading to renal fibrosis.

Our preliminary in vitro experiments verified that ADPN was able to improve oxidative injury caused by high glucose in mesangial cells (19), therefore, in the present study, an ADPN eukaryotic expression vector was injected into DM rats. The pathology information after 12 weeks of injection indicated that the hypertrophy of the kidney was reduced, ECM accumulation had decreased, mesangial expansion had decreased, glomerular sclerosis was less severe, blood sugar and glycosylated hemoglobin levels had decreased, and that extra protein in the urine was markedly decreased. In the present study, it was also revealed that the ROS levels in the renal tissues of the rats in the DM group were higher and the eNOS expression levels were lower than those in the normal control group. For the gAd transfection group, although the ROS levels were higher than those in the normal control group, they was significantly lower than those in the DM group, and the eNOS expression levels were also higher than those in the DM group. Since eNOS was closely associated with the antioxidant capacity of the kidneys and with endothelial protection, it was highly possible that the renal protective effects of ADPN were associated with its antioxidant effects. In addition, the TGF- $\beta 1$ expression levels were higher in the DM group. However, following 12 weeks of APDN injection, the levels were significantly reduced along with the reduction of mesangial areas, which indicated 
that, besides the anti-oxidation mechanisms, APDN could also protect the kidney by reducing the expression of TGF- $\beta 1$ to decrease the accumulation of ECM.

ADPN is hypothesized to function through its specific receptors and different signaling pathways, however, there are few studies in this area. By interacting APDN or gAcrp30 with C2C12 myocytes, Yamauchi et al (20) showed that the AMPK and acetyl coenzyme carboxylase levels increased, and that the effects were more significant with $\mathrm{C} 2 \mathrm{C} 12$ myocytes expressing adiponectin receptor 1 (AdipoR1), which indicated that with the binding to AdipoR1, ADPN was able to activate AMPK. AMPK is a protein kinase presented in the tissues of the majority of mammals. Activated AMPK is able to increase glucose entry, the oxidation of fatty acids and insulin sensitivity. Yamauchi et al (20) suggested that the signal transduction mechanism of APDN was based on the activation of AMPK. ADPN, as an activator of AMPK, is able to activate AMPK in skeletal muscle cells and liver cells, resulting in lower BG levels. The injection of $75 \mu \mathrm{g}$ ADPN into C57BL/6J mice, led to an increase in the activity of AMPK within 15-30 min. Either in vivo or in vitro, the activation of AMPK was the first effect of ADPN. The present study indicated that following activation by one dose of gAd to DM rats, the expression of p-AMPK was promoted in a time-dependent manner, and further experiments indicated that the expression of p-AMPK was lower in the DM group than that in the control group, and that the p-AMPK protein levels in the gAd transfection group were significantly higher. It was verified by statistical analysis that p-AMPK and the protein expression of eNOS were significantly correlated, which indicated that the mechanism could be that AMPK was activated by APDN, which is phosphorylated and further activates eNOS, inhibiting the formation of ROS and protecting the kidneys. The reason that the expression of p-AMPK in the DM group was lower than that in the normal control group may be associated with disease progression in patients with DN.

In summary, the present study demonstrated that the delivery of exogenous gAd attenuates DN, a devastating illness with great morbidity and mortality. ADPN has a protective function in reducing albuminuria, anti-oxidative stress and the expression of TGF- $\beta 1$, and in enhancing the protein expression of eNOS, partly via the AMPK pathway. However, in view of the controversy pertaining to the effects of ADPN on DN in the literature, further studies are required in this area prior to considering its therapeutic utilization in a clinical setting.

\section{References}

1. Stojiljkovic L and Behnia R: Role of renin angiotensin system inhibitors in cardiovascular and renal protection: a lesson from clinical trials. Curr Pharm Des 13: 1335-1345, 2007.
2. Hotta K, Funahashi T, Arita Y, Takahashi M, Matsuda M, Okamoto Y, et al: Plasma concentrations of a novel, adipose-specific protein, adiponectin, in type 2 diabetic patients. Arterioscler Thromb Vasc Biol 20: 1595-1599, 2000.

3. Shimabukuro M, Higa N, Asahi T, Oshiro Y, Takasu N, Tagawa T, et al: Hypoadiponectinemia is closely linked to endothelial dysfunction in man. J Clin Endocrinol Metab 88: 3236-3240, 2003.

4. Arita Y,Kihara S, Ouchi N, Takahashi M, Maeda K, Miyagawa J, et al: Paradoxical decrease of an adipose-specific protein, adiponectin, in obesity. Biochem Biophys Res Commun 257: 79-83, 1999.

5. Lindsay RS, Funahashi T, Hanson RL, Matsuzawa Y, Tanaka S, Tataranni PA, et al: Adiponectin and development of type 2 diabetes in the Pima Indian population. Lancet 360: 57-58, 2002.

6. Kumada M, Kihara S, Sumitsuji S, Kawamoto T, Matsumoto S, Ouchi $\mathrm{N}$, et al: Association of hypoadiponectinemia with coronary artery disease in men. Arterioscler Thromb Vasc Biol 23: 85-89, 2003.

7. Adamczak M, Wiecek A, Funahashi T, Chudek J, Kokot F and Matsuzawa Y: Decreased plasma adiponectin concentration in patients with essential hypertension. Am J Hypertens 16: 72-75, 2003.

8. Holst JJ and Binderup M: Fatty tissue and insulin resistance: resistin and adiponectin. Ugeskr Laeger 164: 2173-2176, 2002 (In Danish).

9. Lin J, Hu FB and Curhan G: Serum adiponectin and renal dysfunction in men with type 2 diabetes. Diabetes Care 30: 239-244, 2007

10. Koshimura J, Fujita H, Narita T, et al: Urinary adiponectin excretion is increased in patients with overt diabetic nephropathy. Biochem Biophys Res Commun 316: 165-169, 2004.

11. Saito T, Saito O, Kawano T, et al: Elevation of serum adiponectin and CD146 levels in diabetic nephropathy. Diabetes Res Clin 78: 85-92, 2007.

12. Sharma K, Ramachandrarao S, Qiu G, et al: Adiponectin regulates albuminuria and podocyte function in mice. J Clin Invest 118: 1645-1656, 2008.

13. Guo X, Liu Z, Li H, et al: A novel rat model of type 2 diabetes mellitus. Chinese Journal of Nephrology Dialysis \& Transplantation 9: 351-355, 2000.

14. Adamczak M, Chudek J and Wiecek A: Adiponectin in patients with chronic kidney disease. Semin Dial 22: 391-395, 2009.

15. Bhatia S, Shukla R, Venkata Madhu S, et al: Antioxidant status, lipid peroxidation and nitric oxide end products in patients of type 2 diabetes mellitus with nephropathy. Clin Biochem 36: 557-562, 2003.

16. Obrosova IG, Fathallah L, Liu E, et al: Early oxidative stress in the diabetic kidney: effect of DL-alpha-lipoic acid. Free Radic Biol Med 34: 186-195, 2003.

17. Park IS, Kiyomoto H, Abboud SL, et al: Expression of transforming growth factor- $\beta$ and type IV collagen in early streptozotocin-induced diabetes. Diabetes 46: 473-480, 1997.

18. Wahab NA, Harper K and Mason RM: Expression of extracellular matrix molecules in human mesangial cells in response to prolonged hyperglycemia. Biochem J 316: 985-992, 1996.

19. Yuan F, Liu F, Liu YH, et al: Effect of adiponectin on reactive oxygen species and endothelial nitric oxide synthase expression induced by high glucose in human mesangial cells. Chin J Nephrol 9: 725-727, 2009.

20. Yamauchi T, Kamom J, Ito Y, et al: Cloning of adiponectin receptors that mediate antidiabetic metabolic effects. Nature 423: 762-769, 2003. 